\title{
USING OBS TO CREATE VIDEO LECTURES FOR ONLINE COUNSELING STUDENTS
}

\author{
Daniel A. Kaufmann, Grand Canyon University
}

\section{ABSTRACT}

Online learners face a different set of obstacles than their classroom-based counterparts as they seek to develop clinical skills solely through interaction with the content of an independent learning curriculum. While ground campus learners benefit from classroom content such as lectures or roleplay activities with their peers to rehearse clinical abilities, online students may struggle to identify ways to connect with the source material in a manner that leads to becoming a professional counselor confident in their own theoretical orientation and the related skillset. To this point, some information is available to explain the impact on using video lectures to allow for class time to experience a flipped classroom format. However, this does not extend to online learners who neither get the benefit of an in-person lecture or counseling related experiential activities during class time. This reflection will seek to bridge this gap, explain how to use software commonly used for streaming video games to make the online class more vibrant, and extend additional learning opportunities to students early in the counseling curriculum who may not have identified their flow yet as an independent learner.

\section{INTRODUCTION}

Online education for counseling students is often structured with a balance between synchronous and asynchronous approaches for learning, which effectively meet the standards for online education standards according to most accreditation bodies (Benshoff \& Gibbons, 2011; Cicco, 2013). Synchronous methods of course participation involve the points in time where the students are interacting in real-time with either their instructor or other students. These can consist of text or video chat, lectures being delivered via video conference software, role plays being completed by students together using technology embedded into the Learning Management System (LMS), and many other approaches that allow the online environment to simulate structured class events across the geographical distance of the participants. However, it is also commonplace for asynchronous methods of course engagement to outweigh the synchronous methods due to the limited resource of the instructor, the schedules of the students being less structured than a traditional graduate student who is attending class at consistent times each week (Gillett-Swan, 2017), and the ease or flexibility of utilizing the LMS as the primary tool for course communications, experience, and learning assessment (Benshoff \& Gibbons, 2011). The inherent challenge of approaching counseling courses this way is that the development of skills and interpersonal traits within the student is critical for the growth of the student to avoid being stifled (Hurst, Cleveland-Innes, Hawranik, \& Gauvreau, 2013). It would seem beneficial for the students in online course environments to address this form of growth in a manner which effectively prepares them from the assumed difficulties of surviving in the field (Cicco, 2013).

In establishing what form of difficulties this study seeks to overcome, it is important to establish types of learners and the various forms of structures online programs can take. First, integration of online formats into classrooms can be done for traditional classrooms using methods which grant learners additional opportunities to wrestle with and retain course content (Gillett- 
Swan, 2017). These can be referred to as "internal learners," since the course content is being delivered in a location that they attend and sit within on a consistent basis. Next, students can participate in the program entirely online. There is no classroom they experience, and all reading of content and attempted informational retention becomes their individual task to complete in isolation from a traditional classroom environment. These can be referred to as "external learners." This concept became clearer to me immediately after completing a course as the instructor in a traditional campus classroom that I had already taught for years in the online format for the same university. The research question that prompted this paper became, "How do I give my external learners the same richness of information and the experience of learning from me as a counselor educator as my traditional students just received?" In reviewing this question, it became clear the topics of conversation with the internal students was often more focused on different topics than those of the external learners. Internal learners were more prone to explore the further development of that connection with me as their instructor to more fully understand the content being delivered to them. By contrast, the external learners' dialogue was much more likely to revolve around the number of points being awarded in my evaluation of their work. This often led to a higher focus on the interpretation of course deadlines rather than more beneficial elements of student learning such as course philosophy, skill development, or application of counseling work in real life scenarios.

Building off this observation, the consistent positive qualities of the faculty-student interactions with internal learners was viewed in stark contrast to the complexities of providing feedback to the external learners. While research on online learning does indicate some insights into the role of human development and negative self-evaluations for students receiving negative feedback (or even gatekeeping efforts) from their course instructor (Stroud, Olguin, \& Marley, 2016), these differences felt to me more related to the experienced delivery of content as a means of structuring the view of the student related to themselves connected to the task of learning the profession of counseling. In the context of counseling and psychology, the "grading as a teaching moment" structure of online feedback delivery began hurting the relationship between me and my external learners in a way similar to the selection of surrogate object amongst the monkeys in the Harlow experiments of the 1950's (Vicedo, 2009). Essentially, experiencing a positive state is more valuable to a living organism than uncomfortably having a basic need met. The goal for my external learners became to record my lectures in an effort to deliver new teaching moments for them into the asynchronous structures of the course and enhance the perception of me as an educator beyond my reductionist role of merely being the member of the course authorized to submit grades.

\section{Using OBS as a Lecture Creating Software}

The solution this paper will discuss was to use Open Broadcaster Software (OBS) as a means of recording my lectures in the same way video games get streamed on websites such as Twitch or Mixer. However, those game streaming sites would be considered synchronous, as the gamer is streaming their gameplay to their audience in real-time, and all interactions continue accordingly until the conclusion of the broadcast. Knowing this would likely not work due to the unknown quality of my external learners' readiness to attend such a lecture event as a stream, I knew it would be more effective to create a resource which could be utilized by my students asynchronously, the same as most other resources in the structure of my university's LMS. Additionally, we do not live in a world where educators of higher learning institutions normally stream lectures as an entertainment medium, so such websites are not normally explored by people for this form of content.

\section{Downloading and Understanding the Software}

The first step is to download the Open Broadcaster Software program (https://obsproject. $\mathrm{com} /$ ). This is a free program developed for people to be able to stream content onto Internet webpages such as Twitch or YouTube without investing quantities of money into their online project. Upon opening the program and setting it up for either streaming (complex set-up) or recording-only (simple setup), the view in Figure 1 is reflected on your computer screen. The initial goal once the program is open is to familiarize yourself with the basic setup of how this program becomes an online 
lecture environment. As noted in Figure 1, there are three sections of the program that are important to understand in order to capitalize on the purpose of this paper:

- The Presentation Window

- The Scenes List

- The Sources List

Once you can identify all three sections on the control panel, you will be able to move on to the next steps in organizing the display for your online lecture video.

The next phase of the OBS lecture setup involves using each of the three panels you identified in step 1. The Scenes List allows for the creation of different backgrounds that can be alternated between by selecting them from the list. For the purpose of this article, only the creation of one scene is necessary. The Presentation Window is like an interactive PowerPoint slide, which you will set up for use as the video display. This rectangle space will display every item in the scene, which is entered to the Sources List. The Sources List is a place where each element, image, text display, and image from the webcam will be displayed in the location it is set at by the course instructor. This is the most time-consuming element of programming in your lecture space into OBS, but it also provides a depth of creative opportunity depending on the vision of the presenter to create the visual experience of the lecture.

To begin working with the Sources List, it is important to understand how the items displayed under the + sign are used to create the lecture space. Upon selecting the plus sign to add an item to your display screen, you will see a list of options. The main items you will need to complete this lecture task are the following:

- Image

- Video Capture Device

- Display Capture

- Text (GDI+)

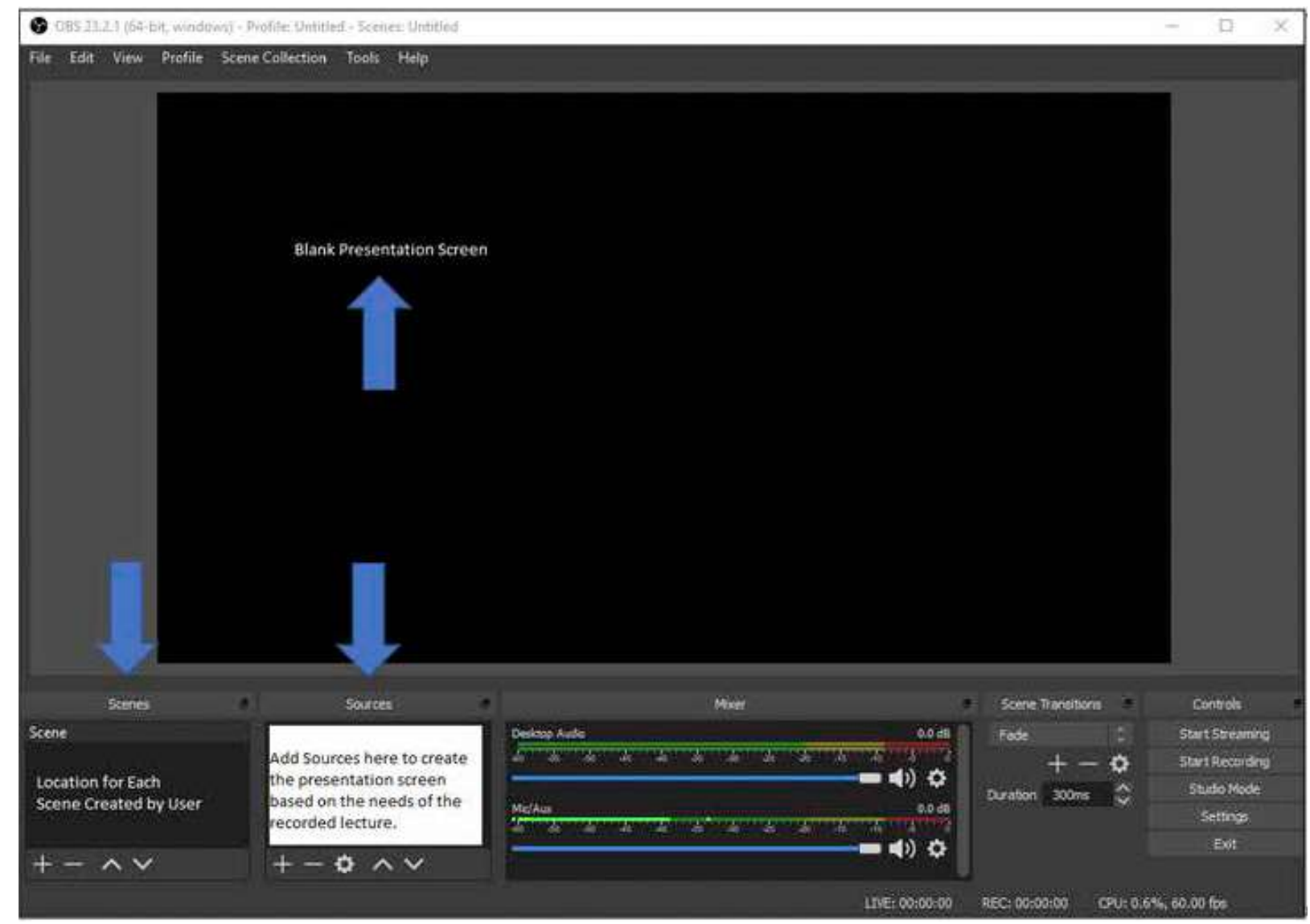

Figure 1. Starting Screen for Open Broadcaster Software 
The first thing I select is the Image item. Once selected, an additional box will ask you to name the object for your Sources list. Next, a prompt will ask you to select an image file from your computer to place in the display environment. For lecture purposes, I use an image that is wallpaper sized and displays my university logo in the upper left. This leaves me appropriate space to add other items to the source list and place them in a way that allows me to display what is needed to support the lecture video.

The second item needed is the webcam source. After selecting the "add source" button, choose "Video Capture Device." OBS will ask you to select your webcam device and will then allow you to move and slide the size of this video box to a location on the display environment that fits your vision for the lecture video. I choose to put this off to the side, so the focus is still on the PowerPoint slides. These will be added in the next step.

To add the PowerPoint to the display environment, add Display Capture to the Sources list. It should be noted that this project does require there to be a dual-screen setup for the PC for the easiest process. This allows for the OBS program to run on the main screen, and the PowerPoint can be presented using the second monitor while being streamed onto the display area of the first monitor. Once the PowerPoint monitor is added to the Sources List, again slide and adjust the window to the desired space in the presentation environment. Once this has been added, everything else comes down to personal preference and creating the desired appearance to your presenter screen.

One valuable addition that can help create the intended appearance for the lecture video is to understand the use of image files to create borders for source items and remembering to utilize the Text $(\mathrm{GDI}+)$ item type to add information to the display. To add borders, I recommend searching for "streaming borders" on the Internet and focusing on finding files with a checkered background that can save as a PNG image file (.png). The checkerpattern indicates it should be transparent when you use the image in OBS. For the image in Figure 2, silver borders are used for the "Required Reading" box and for the PowerPoint presentation, while a "Presenter Border" is placed around the webcam box. These are selected to go well with the purple background and other visual elements present on the screen. Other borders are available (even for free) across the Internet with proper searching. This section relies on the lecturer having a vision for what they would like the video to look like as a finished product for their online class.

There are other items visible in Figure 2 that can be briefly explained for use in this article as well. The "Required Reading" and "Inspirational Quote" items are included to utilize the screen real estate in ways which can still be useful for the student. The text is generated by hitting to add a source, selecting Text (GDI+), and then typing the intended message and selecting the font and other text options. Once "OK" has been selected, the text can be dragged to the proper location on the presenter screen. A silver border is placed here, along with a black background image and an image of the textbook. These are saved on the PC as separate image files and added to the screen using the Sources List. The inspirational quote depicted is created using the same text option and a black background image dragged into a rectangular shape, and then having the opacity of the square altered to make it more transparent as a background for the quote under the lecture portion of the screen.

One final tip involves changing the dimensions of the square objects on the screen. When dragging the sides and corners by their red dots, holding down the "shift" key allows for the side being moved to change the ratio of that edge to the rest of the shape. This is important when setting up objects together to make sure they end in proper alignment with each other.

Once the screen is set, the faculty member will hit "Start Recording" and deliver the lecture. Once completed, simply click the "Stop Recording" button, and the default location for the video file will be found in the "Videos" folder located in "File Explorer" (using Windows 10). With the video file saved to the PC, the file can then be uploaded to a website such as YouTube. If the video settings are set to "Public," then everyone who finds your page will be able to view it. Setting this option to "Unlisted" instead allows only those people who have the link to view the video, but general Internet searching will not show this video to others. This allows the course instructor to control access to the videos so they are watched at the intended times during the course and only by people who should have access to them. 


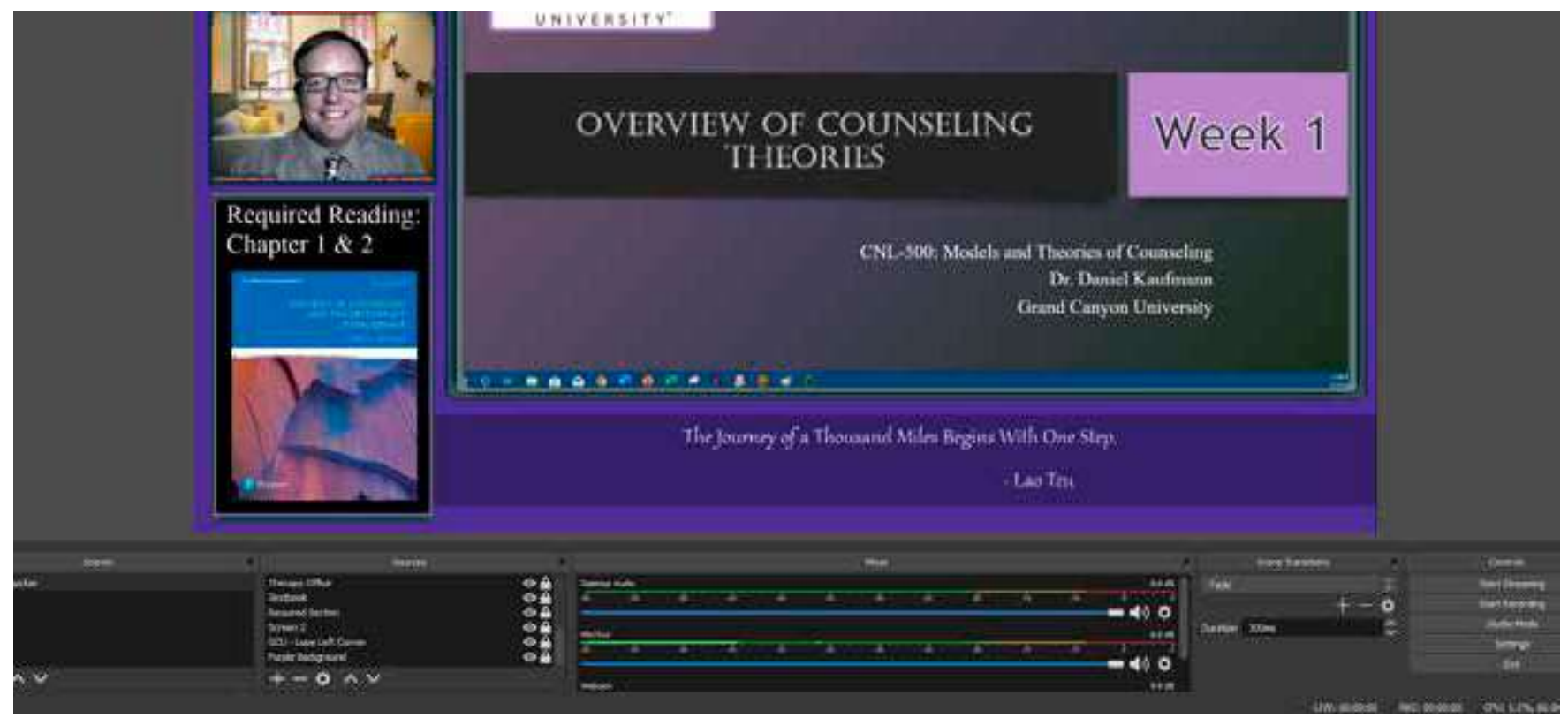

Figure 2.Example of an OBS Setup for Online Lectures

\section{Improvements to the Online Learning Process}

Through utilizing OBS to create a lecture resource for external learners, an important element of classroom learning is added to the online environment. While the curriculum for both in-person and online formats is meant to balance the opportunities for didactic and experiential learning opportunities (Benshoff \& Gibbons, 2011), online may skew more easily into the isolated/selfdirected learning style. Allowing for the presence of faculty lectures in an online classroom adds a necessary element of collaborative learning back into the counseling course experience. While this approach opens up the opportunity to establish a flipped classroom model for in-person classrooms (Long, Logan, \& Waugh, 2016), the video lectures open a dialogue between students and faculty to overcome misconceptions the student may have or confusion related to the course content.

The structure of faculty goals in the counseling program in which the author of this article teaches, focuses on a few critical tasks to guide the online learners through their goals. This list includes daily forum interaction, responding to typed and recorded questions from students, and providing assignment feedback. This list is shorter than the list of a traditional faculty, which has all the same tasks, but includes planning course time, creating lectures, and several other tasks. While this article focuses on the online instructor taking on a parallel process of these campus tasked, it seems reasonable to consider these tasks as equally (if not more) critical to the learning process of a selfdirected learner (Hurst et al., 2013) who is learning sans weekly verbal or collaborative faculty interaction. Consider the following examples of potential student questions from a counseling theories course:

"I am having difficulty understanding this course. It seems like every theory is based on outdated ideas, and I am not relating to the material or the logic of the founding theorists. Would it be okay if I just focus on how to help clients in the next assignment in general rather than trying to use the theories from this week?"

In this example, the student is attempting to understand the theory of counseling but is struggling to embrace the theory in a way which feels usable to them. If the faculty member has prepared an online lecture where they are able to explore the required text while also providing examples from past cases and taking strides to attempt a transformation of the information to feel tangible to the early-developing counseling identity of beginning counseling students, the following example message could just as easily have been presented in the faculty message forum: 
"The lecture video was very helpful and informative. When I was doing the reading this week, I was not sure I was interested in learning further about this theory. However, you explained some of the ideas I basically skipped over in a way that really clicked for me. I see a lot of value in those ideas now, and I wanted to say thank you for spending your time to make this class an awesome learning experience. I have not had a class use the opportunity to lecture this way so far in the program."

Having these resources available also benefits students of all types in the sense that videos can be paused and re-watched, allowing for better note taking and reviewing of concepts the student found critical to their understanding of the topic. Both traditional and online students have commented this resource being available is a new experience that has completely changed how they learn in my course compared to others previously experienced. These transformations to course dialogue represent a notable shift in the collaborative opportunity for both the faculty and student in the setting of an online course. For this reason, it is hoped that software such as OBS can be creatively interacted with by online educators to begin transforming the medium beyond the usual limitations of teaching students in this way.

\section{Areas for Growth}

As more programs of higher learning embrace the online model, strengths and limitations to this medium of teaching are sure to be researched (Cicco, 2012; Hurst et al., 2013). One area for growth in relation to counselor education is to ensure the presence of experiential learning and collaboration between students with other students and their faculty members. The development of counseling skills is integral to numerous phases of counselor learning (Kaufmann, 2019; Ronnestad \& Skovholt, 2003). Ensuring that all programs, online and traditional, take adequate time to address creatively the need for communication skill-building and sharing of constructivist opportunities toward learning (Merlin, 2016) is important at this phase in the transition of education. Based on the discussion of the article, counseling programs benefit from ensuring all faculty are provided support for, encouraged to, and potentially required to interact at a higher level than the basic online course with their students. This aids the students in overcoming the hurdles of lacking a philosophical connection at the onset with new conceptual materials, as they are then being presented the course content by a veteran of the field who is assumed to have a passion for the subject they are presenting to their external learners. One final step would be to research beyond the subject of this article, which focuses on using a program external to the online Learning Management Systems. In this line of thinking, it would seem beneficial to seek out software that can be integrated directly into the system.

\section{CONCLUSION}

It is possible for online faculty to address the inherent obstacles online learners face to facilitate learning via lecture if desired. While ground campus learners simply attend their classroom content in person, online students are expected to learn via independent learning, and in some cases their own trial and error. To this point, some information is available to explain the impact on using video lectures to allow for class time to experience a flipped classroom format. By using software creatively, the format of student-to-faculty interaction can still lead to collaborative learning opportunities. Through articles such as these, it is becoming apparent that Web 2.0 tools can improve class experiences and course outcomes. All it takes is the desire from both student and faculty to create the learning environment they desire, and to approach this preference with the creativity to reject limitations and find a way to make the education experience look as close as possible to what their ideal approach would encompass. 


\section{References}

Benshoff, J. M., \& Gibbons, M. M. (2011). Bringing life to e-Learning: Incorporating a synchronous approach to online teaching in counselor education. Professional Counselor, 1(1), 21-28.

Cicco, G. (2012). Counseling instruction in the online classroom: A survey of student and faculty perceptions. Journal on School Educational Technology, 8(2), 1-10.

Cicco, G. (2013). Online course effectiveness: A model for innovative research in counselor education. Journal on School Educational Technology, 9(1), 10-16.

Gillett-Swan, J. (2017). The challenges of online learning supporting and engaging the isolated learner. Journal of Learning Design, 10(1), 20-30.

Hurst, D., Cleveland-Innes, M., Hawranik, P., \& Gauvreau, S. (2013). Online graduate student identity and professional skills development. Canadian Journal of Higher Education, 43(3), 36-55.

Kaufmann, D. A. (2019). Counselor development as the hero's journey: Reflections from a counselor educator. Journal of Instructional Research, 8, 17-32.

Long, T., Logan, J., \& Waugh, M. (2016). Students' perceptions of the value of using videos as a pre-class learning experience in the flipped classroom. TechTrends: Linking Research \& Practice to Improve Learning, 60(3), 245-252. doi:10.1007/ s11528-016-0045-4

Merlin, C. (2016). Flipping the counseling classroom to enhance application-based learning activities. Journal of Counselor Preparation \& Supervision, 8(3), 44.

Ronnestad, M. H., \& Skovholt, T. M. (2003). The journey of the counselor and therapist: Research findings and perspectives on professional development. Journal of Career Development, 30(1), 5-44.

Stroud, D., Olguin, D., \& Marley, S. (2016). Relationship between counseling students' childhood memories and current negative self-evaluations when receiving corrective feedback. International Journal for the Advancement of Counselling, 38(3), 237-248.

Vicedo, M. (2009). Mothers, machines, and morals: Harry Harlow's work on primate love from lab to legend. Journal of the History of the Behavioral Sciences, 45(3), 193-218. doi:10.1002/jhbs. 20378 